PROCEEDINGS OF THE

AMERICAN MATHEMATICAL SOCIETY

Volume 135, Number 4, April 2007, Pages 1151-1157

S 0002-9939(06)08585-6

Article electronically published on October 13, 2006

\title{
COMPACTNESS PROPERTIES OF OPERATORS DOMINATED BY AM-COMPACT OPERATORS
}

\author{
BELMESNAOUI AQZZOUZ, REDOUANE NOUIRA, AND LARBI ZRAOULA
}

(Communicated by Joseph A. Ball)

\begin{abstract}
We study several properties about the problem of domination in the class of positive AM-compact operators, and we obtain some interesting consequences on positive compact operators. Also, we give a sufficient condition under which a Banach lattice is discrete.
\end{abstract}

\section{INTRODUCTION}

An interesting problem in the operator theory on Banach lattices is that of finding conditions under which properties of a positive operator $T$ will be inherited by any positive operator smaller than (or dominated by) $T$. In other words, if $E$ and $F$ are two Banach lattices and $S, T$ are two operators from $E$ into $F$ such that $0 \leq S \leq T$, we have to study conditions on $E$ and $F$ under which a nice property of $T$ will be inherited by $S$.

For compact operators, this problem was studied by Dodds-Fremlin 8], Aliprantis-Burkinshaw [1, Wickstead [16, 17] and Aqzzouz-Nouira [7. The domination problem for weakly compact operators was studied simultaneously by Aliprantis-Burkinshaw [2] and Wickstead [15. For Dunford-Pettis operators, this problem was studied by Aliprantis-Burkinshaw 3, Kalton-Saab 13 and Wickstead [16. Also, Flores-Hernandez studied the domination problem for disjointly strictly singular operators [9] and strictly singular operators [10. Finally, Flores-Ruiz [11] obtained some interesting results for the class of narrow operators.

The problem of domination in the class of positive AM-compact operators was originally studied by Fremlin in [12]. He showed that if the norm of $F$ is order continuous, then the subspace of all AM-compact operators from $E$ into $F$ is a band (i.e. an order ideal which is order closed). Recall from Zaanen [18, that a regular operator $T$ from a vector lattice $E$ into a Banach lattice $F$ is said to be AM-compact if it carries each order bounded subset of $E$ onto a relatively compact subset of $F$.

Our objective in this paper is to continue the investigation of the domination problem for the class of AM-compact operators. First, we will prove that the second power of an AM-compactly dominated operator is always AM-compact. Also, we shall give a necessary and sufficient condition for when the AM-compactness of a positive operator which is AM-compactly dominated is inherited. As a consequence,

Received by the editors July 7, 2005 and, in revised form, November 14, 2005.

2000 Mathematics Subject Classification. Primary 46A40, 46B40, 46B42.

Key words and phrases. AM-compact operator, order continuous norm, discrete vector lattice. 
we will obtain some interesting and well-known properties on the domination problem for positive compact operators (Theorem 2.2 of [1] and Theorem 2.1 of [7]). Finally, we will give a sufficient condition under which the topological dual of a Banach lattice is discrete.

\section{MAJOR RESUlts}

A subset $D$ of a Banach lattice $E$ is said to be almost order bounded if for each $\varepsilon>0$, there exists some $x \in E^{+}$such that $D \subset[-x, x]+\varepsilon B_{E}$, where $B_{E}$ is the unit ball of $E$. An operator $T$ from a Banach space $E$ into a Banach lattice $F$ is said to be semi-compact if it maps bounded subsets of $E$ onto almost order bounded subsets of $F$. The class of semi-compact operators fails to satisfy the duality problem, but it follows from Theorem 18.20 of [4, that the class of semicompact operators satisfies the domination property. For unexplained terminology on Banach lattice theory, we refer to Zaanen [18.

It was well known that if $S$ and $T$ are operators from $E$ into $E$ such that $0 \leq S \leq T$ and $T$ is AM-compact and L-weakly compact (resp. M-weakly compact), then $S^{2}$ is compact (4], Exercice 12 (resp. Exercice 13), p. 331). The following evident proposition gives a similar result.

Proposition 2.1. Let $E$ be a Banach lattice, and let $S$ and $T$ be operators from $E$ into $E$ such that $0 \leq S \leq T$ with $T$ semi-compact and $S A M$-compact. Then $S^{2}$ is compact.

Proof. In fact, if $T$ is semi-compact, then $S$ is too (Theorem 18.20 of [4]), and since $S$ is AM-compact, then the second power operator $S^{2}$ is compact.

If $E^{\prime}$ is the topological dual of $E$, the absolute weak topology $|\sigma|\left(E, E^{\prime}\right)$ is the locally convex solid topology on $E$ generated by the family of lattice seminorms $\left\{P_{f}: f \in E^{\prime}\right\}$, where $P_{f}(x)=|f|(|x|)$ for each $x \in E$. Similarly, $|\sigma|\left(E^{\prime}, E\right)$ is the locally convex solid topology on $E^{\prime}$ generated by the family of lattice seminorms $\left\{P_{x}: x \in E\right\}$, where $P_{x}(f)=|f|(|x|)$ for each $f \in E^{\prime}$. For more information, we refer the reader to $[5]$.

We will need the following lemma which is a consequence of a Grothendieck's Theorem ([14], Theorem 3, p. 51).

Lemma 2.2. Let $E$ and $F$ be two Banach lattices and let $T: E \longrightarrow F$ be an operator. Then for each $x \in E^{+}, T([-x, x])$ is norm precompact in $F$ if and only if $T^{\prime}\left(B_{F^{\prime}}\right)$ is precompact for $|\sigma|\left(E^{\prime}, E\right)$ in $E^{\prime}$.

Without any conditions on the Banach lattice, we have the following result.

Theorem 2.3. Let $E$ be a Banach lattice and let $S$ and $T$ be two operators from $E$ into $E$ such that $0 \leq S \leq T$ and $T$ is AM-compact; then $S^{2}$ is AM-compact.

Proof. Let $x \in E^{+}$; then $T([0, x])$ is norm precompact in $E$. Since the topology defined by the norm of $E$ is finer than $|\sigma|\left(E, E^{\prime}\right)$, then $T([0, x])$ is precompact for $|\sigma|\left(E, E^{\prime}\right)$. An application of Lemma 1.1 of $[3]$ implies that $S([0, x])$ is precompact for $|\sigma|\left(E, E^{\prime}\right)$. It now follows from Theorem 1.2 of [1] that $S^{\prime}([0, f])$ is precompact for $|\sigma|\left(E^{\prime}, E\right)$ for each $f \in\left(E^{\prime}\right)^{+}$. Hence $S^{\prime}$ maps almost order bounded subsets of $E^{\prime}$ onto precompact subsets for $|\sigma|\left(E^{\prime}, E\right)$.

On the other hand, if $B_{E^{\prime}}^{+}$denotes $B_{E^{\prime}} \cap\left(E^{\prime}\right)^{+}$, then $S^{\prime}\left(B_{E^{\prime}}^{+}\right)$is almost order bounded. It follows that $\left(S^{\prime}\right)^{2}\left(B_{E^{\prime}}^{+}\right)$is precompact for $|\sigma|\left(E^{\prime}, E\right)$. Now, Lemma 
2.2 implies that $S^{2}([0, x])$ is norm relatively compact for each $x \in E^{+}$. Therefore $S^{2}$ is AM-compact.

By using the same arguments as in Example 3.2 of [1], we obtain the following consequence.

Corollary 2.4. Let $E, F$ and $G$ be Banach lattices. Let $S_{1}, T_{1}: E \longrightarrow F$ and $S_{2}, T_{2}: F \longrightarrow G$ be operators such that $0 \leq S_{i} \leq T_{i}$ and each $T_{i}$ is AM-compact, $i=1,2$. Then $S_{2} S_{1}$ is an AM-compact operator.

Let us recall that a vector lattice $E$ is said to be order complete if each nonempty subset that is bounded from above has a supremum. Another consequence is the following result.

Corollary 2.5. Let $E$ be an order complete Banach lattice. If $T$ is a regular operator such that $|T|$ is AM-compact, then the second power operator $T^{2}$ is $A M$ compact.

Proof. In fact, $(T)^{2}=\left(T^{+}-T^{-}\right)^{2}=\left(T^{+}\right)^{2}-T^{+} T^{-}-T^{-} T^{+}+\left(T^{-}\right)^{2}$ with $0 \leq$ $T^{+} \leq|T|, 0 \leq T^{-} \leq|T|$ and $|T|$ is an AM-compact operator. The assertion follows immediately from Theorem 2.3 and Corollary 2.4.

Remark 2.6. There exist Banach lattices $E$ and $F$ and there exist positive operators $S$ and $T$ from $E$ into $F$ such that $0 \leq S \leq T$, with $T$ being AM-compact but with $S$ not being AM-compact. In fact

Examples 2.7. 1. Let $S_{1}, T_{1}, S_{2}$ and $T_{2}$ be the positive operators defined in Example 3.1 of 1 . We have $0 \leq S_{i} \leq T_{i}$ for $i=1,2$, and each $T_{i}$ is compact. In 1, it was proved that $S_{2} S_{1}$ is not compact. We have to show that $S_{2}$ is not AM-compact. If not, since $0 \leq S_{1} \leq T_{1}$ and the operator $T_{1}$ is semi-compact, then $S_{1}$ is semi-compact (Theorem 18.20 of [4]). Finally, $S_{2} S_{1}$ is a compact operator as a product of a semi-compact operator with an AM-compact operator. But this is impossible.

2. Let $E$ be the Banach lattice $l^{1} \oplus L^{2} \oplus l^{\infty}$ and let $S, T: E \longrightarrow E$ be the operators defined in [1, Example 3.2, where $T_{1}, T_{2}, S_{1}$ and $S_{2}$ are the operators of the above example. It is clear that $0 \leq S \leq T$ and $T$ is AM-compact but $S^{2}$ is not compact, and hence $S$ is not AM-compact.

Remarks 2.8. 1. If $R, S$ and $T$ are operators from $E$ into $E$ such that $R \leq S \leq T$ and $R, T$ are AM-compact, then $S^{2}$ is a AM-compact operator. In fact, since $0 \leq S-R \leq T-R$ and $T-R$ is an AM-compact operator, the second power operator $(S-R)^{2}=S^{2}-S R-R S+R^{2}$ is AM-compact. The result follows.

2. If $E$ is an infinite-dimensional AM-space with unit, there is no positive AMcompact operator $T$ on $E$ such that $0 \leq I d_{E} \leq T$, where $I d_{E}$ is the identity operator of $E$. In fact, whenever $E$ is an AM-space with unit, the class of AMcompact operators on $E$ coincides with the class of regular compact operators on E.

Recall that a nonzero element $x$ of a vector lattice $E$ is discrete if the order ideal generated by $x$ equals the subspace generated by $x$. The vector lattice $E$ is discrete if it admits a complete disjoint system of discrete elements.

A norm \|\| of a Banach lattice $E$ is order continuous if for each net $\left(x_{\alpha}\right)$ such that $x_{\alpha} \downarrow 0$ in $E$, the sequence $\left(x_{\alpha}\right)$ converges to 0 for the norm \|\| , where the 
notation $x_{\alpha} \downarrow 0$ means that the sequence $\left(x_{\alpha}\right)$ is decreasing, its infimum exists and $\inf \left(x_{\alpha}\right)=0$.

Recall that if $S$ and $T$ are operators from $E$ into $E$ satisfying $0 \leq S \leq T$ such that $T$ is L-weakly compact and AM-compact, then $S$ is AM-compact (Exercice 12 of 4, p. 331). The following result gives a sufficent condition on the Banach lattice, under which the AM-compactness of a positive operator $T$ will be inherited by any positive operator smaller than $T$.

Proposition 2.9. Let $E$ and $F$ be Banach lattices and let $S$ and $T$ be operators from $E$ into $F$ such that $0 \leq S \leq T$ and $T$ is $A M$-compact. If for each $x \in E^{+}$the vector lattice $\left(E_{x}\right)^{\prime}$ is discrete, then the operator $S$ is AM-compact.

Proof. Let $S$ and $T$ be operators from $E$ into $F$ such that $0 \leq S \leq T$ and $T$ is AM-compact. It is clear that $S$ is AM-compact if and only if for each $x \in E^{+}$, the restriction $S_{\mid E_{x}}$ from $E_{x}$ into $F$ is compact, where $E_{x}$ is the order ideal generated by $x$. Since $T_{\mid E_{x}}$ from $E_{x}$ into $F$ is compact, $0 \leq S_{\mid E_{x}} \leq T_{\mid E_{x}}$ and $\left(E_{x}\right)^{\prime}$ is discrete with an order continuous norm, it follows from Theorem 1 of [16] that $S_{\mid E_{x}}$ is compact. This proves the result.

The following theorem gives a necessary and sufficient condition for which the domination problem admits a positive solution for the class of positive AM-compact operators.

Theorem 2.10. Let $E$ and $F$ be Banach lattices. Then the following statements are equivalent:

1) For all operators $S, T: E \longrightarrow F$ such that $0 \leq S \leq T$ and $T$ is AM-compact, the operator $S$ is AM-compact.

2) One of the following conditions holds:

i. The norm of $F$ is order continuous.

ii. The topological dual $E^{\prime}$ is discrete.

Proof. The implication $i \Longrightarrow 1$ is just a theorem of Fremlin [12].

For the implication $i i \Longrightarrow 1$ let $S$ and $T$ be operators from $E$ into $F$ such that $0 \leq S \leq T$ and $T$ is AM-compact. Then for each $x \in E^{+}, T([0, x])$ is norm precompact in $F$, and hence $T^{\prime}\left(B_{E^{\prime}}\right)$ is precompact for $|\sigma|\left(E^{\prime}, E\right)$ (Lemma 2.2). Since $0 \leq S^{\prime} \leq T^{\prime}$, it results from Theorem 3.1.b of [6] that $S^{\prime}\left(B_{F^{\prime}}\right)$ is also precompact for $|\sigma|\left(E^{\prime}, E\right)$. A second application of Lemma 2.2 gives the result.

$1 \Longrightarrow 2$. Assume that either of the conditions $\mathrm{i}$ and ii is true. Since the norm of $F$ is not order continuous, there exist some $z \in F^{+}$and a disjoint sequence $\left(z_{n}\right)$ in $[0, z]$, which does not admit any subsequence converging to 0 for the norm (Theorem 3.22 of [5]). Also, there exist some $\Phi \in\left(E^{\prime}\right)^{+}$and a sequence $\left(\Phi_{n}\right)$ in $[0, \Phi]$, which converges to 0 for the weak topology $\sigma\left(E^{\prime}, E\right)$ but does not converge to 0 for the absolute weak topology $|\sigma|\left(E^{\prime}, E\right)$ (Corollary 6.57 of [5]). This implies that there exists some $y \in E^{+}$and a sequence $\left(y_{n}\right)$ in $[0, y]$ such that $\Phi_{n}\left(y_{n}\right)=1$ for each $n \in \mathbb{N}$.

Let $\hat{E}$ be the completion of $E$ for the absolute weak topology $|\sigma|\left(E, E^{\prime}\right)$, and let $P_{n}$ be the principal projection on the band $B_{n}$ generated by $y_{n}$ in $\hat{E}$. We can assume that $\Phi_{n}\left(y_{m}\right)=0$ if $n \neq m$ (if not, we replace $\Phi_{n}$ by $\Phi_{n} \circ P_{n}$ ).

Let $S$ be the positive operator defined by $S(x)=\left(\sum_{n=1}^{+\infty} \Phi_{n}(x) z_{n}\right)+\Phi(x) z$ for each $x \in E^{+}$. Since $\left(z_{n}\right)$ is a disjoint sequense and $\left(\Phi_{n}\right)$ converges to 0 weakly, the operator $S$ is well defined. 
The operator $S$ is not AM-compact. If not, the sequence $\left(S\left(y_{n}\right)\right)=\left(\Phi\left(y_{n}\right) z+z_{n}\right)$ admits a convergent subsequence that we also denote by $\left(\Phi\left(y_{n}\right) z+z_{n}\right)$. But since the sequence $\left(\Phi\left(y_{n}\right)\right)$ admits a convergent subsequence, it follows that $\left(z_{n}\right)$ admits a convergent subsequence. This presents a contradiction, and hence $S$ is not AMcompact. However the operator $T$ defined by $T(x)=2 \Phi(x) z$ is AM-compact and $0 \leq S \leq T$. This completes the proof.

Now, as a consequence, we obtain Theorem 2.2 of [1] and Theorem 2.1 of [7].

Corollary 2.11. Let $E$ be a Banach lattice. Then for each pair of operators $S$ and $T$ from $E$ into $E$ such that $0 \leq S \leq T$ with $T$ compact, the operator $S^{2}$ is compact if one of the following assertions is valid:

1. The norm of $E$ is order continuous.

2. For each $x \in E^{+},\left(E_{x}\right)^{\prime}$ is discrete.

3. The topological dual $E^{\prime}$ is discrete.

4. The norm of $E^{\prime}$ is order continuous.

Proof. For assertions 1 and 3 (resp. 2) it follows from Theorem 2.10 (resp. Proposition 2.9) that $S$ is AM-compact and an application of Proposition 2.1 implies the assertion.

For assertion 4 , since $0 \leq S^{\prime} \leq T^{\prime}$ and the norm of $E^{\prime}$ is order continuous, the result follows from assertion 1.

Remark 2.12. If $T: E \rightarrow F$ is a bounded operator between two Banach lattices, Aliprantis and Burkinshaw [4] defined the ring ideal Ring $(T)$ generated by $T$ as the norm closure in $L(E, F)$ of the vector subspace consisting of all operators of the form $\sum_{i=1}^{n} R_{i} T S_{i}$, where $S_{i} \in L(E, E)$ and $R_{i} \in L(F, F)$, and where $L(E, F)$ is the Banach space of all norm bounded operators from $E$ into $F$. They proved that if $E=F$ and if $S: E \rightarrow E$ is another operator that satisfies $0 \leq S \leq T$ such that $T$ is compact, then we have:

a) $S^{3} \in \operatorname{Ring}(T)$ (in particular $S^{3}$ is compact).

b) $S^{2}$ belongs to $\operatorname{Ring}(T)$ (in particular $S^{2}$ is compact) whenever $E$ has an order continuous norm.

It is natural to ask if we can obtain similar results for the class of AM-compact operators. Unfortunately, this is not true. In fact,

1) if $S^{2} \in \operatorname{Ring}(T)$ whenever $0 \leq S \leq T$ with $T$ AM-compact, then in particular $S^{2}$ is AM-compact. Hence, the operator $S^{2}$ will be compact whenever $T$ is compact. But this is not true in general.

2) Also, if $S \in \operatorname{Ring}(T)$ whenever $0 \leq S \leq T$ with $T$ AM-compact and the norm of $E$ order continuous or the topological dual $E^{\prime}$ discrete, then in particular $S$ is AM-compact. Hence, under these conditions (i.e. the norm of $E$ is order continuous or the topological dual $E^{\prime}$ is discrete) the operator $S$ will be compact whenever $T$ is compact. But this is false in general.

Finally, the following result gives a sufficent condition under which a Banach lattice is discrete.

Theorem 2.13. Let $E$ be a Banach lattice. If for each $x \in E^{+}$the vector lattice $\left(E_{x}\right)^{\prime}$ is discrete, then the topological dual $E^{\prime}$ is discrete.

Proof. Assume that for each $x \in E^{+},\left(E_{x}\right)^{\prime}$ is discrete. Take $G=E \oplus l^{1} \oplus c$, where $c$ is the Banach lattice of all convergent sequences. Let $S, T: G \longrightarrow G$ be operators 
such that $0 \leq S \leq T$ and $T$ is compact. Each one of our operators is of the form

$$
S=\left(\begin{array}{lll}
a_{1} & b_{1} & c_{1} \\
a_{2} & b_{2} & c_{2} \\
a_{3} & b_{3} & c_{3}
\end{array}\right) \text { and } T=\left(\begin{array}{ccc}
a_{1}^{\prime} & b_{1}^{\prime} & c_{1}^{\prime} \\
a_{2}^{\prime} & b_{2}^{\prime} & c_{2}^{\prime} \\
a_{3}^{\prime} & b_{3}^{\prime} & c_{3}^{\prime}
\end{array}\right)
$$

Hence

$$
S^{2}=\left(\begin{array}{ccc}
a_{1} a_{1}+b_{1} a_{2}+c_{1} a_{3} & a_{1} b_{1}+b_{1} b_{2}+c_{1} b_{3} & a_{1} c_{1}+b_{1} c_{2}+c_{1} c_{3} \\
a_{2} a_{1}+b_{2} a_{2}+c_{2} a_{3} & a_{2} b_{1}+b_{2} b_{2}+c_{2} b_{3} & a_{2} c_{1}+b_{2} c_{2}+c_{2} c_{3} \\
a_{3} a_{1}+b_{3} a_{2}+c_{3} a_{3} & a_{3} b_{1}+b_{3} b_{2}+c_{3} b_{3} & a_{3} c_{1}+b_{3} c_{2}+c_{3} c_{3}
\end{array}\right) .
$$

Since for each $x \in E^{+},\left(E_{x}\right)^{\prime}$ is discrete, $a_{1}: E \longrightarrow E, a_{2}: E \longrightarrow l^{1}$ and $a_{3}: E \longrightarrow c$ are AM-compact. On the other hand, if $l^{1}$ is discrete and its norm is order continuous, then it follows from Theorem 1 of $\left[16\right.$ that $c_{2}: c \longrightarrow l^{1}$, $b_{2}: l^{1} \longrightarrow l^{1}$ and $a_{2}: E \longrightarrow l^{1}$ are compact. Now, since $c^{\prime}$ is discrete and its norm is order continuous, Theorem 1 of [16] implies that $c_{1}: c \longrightarrow E$ and $c_{3}: c \longrightarrow c$ are compact. This shows that $S^{2}$ is a compact operator. Finally, Theorem 1.1 of [7] implies that the norm of $G$ is order continuous or the norm of $G^{\prime}$ is order continuous or the topological dual $G^{\prime}$ is discrete. But the two first conditions are false for our space $G$, hence $G^{\prime}$ is discrete. This proves that $E^{\prime}$ is discrete.

\section{ACKNOWLEDGEMENTS}

The authors thank the referee for his valuable suggestions and remarks concerning the content of this paper.

\section{REFERENCES}

[1] Aliprantis, C.D. and Burkinshaw, O., Positive compact operators on Banach lattices. Math. Z. 174 (1980), 289-298. MR0593826 (81m:47053)

[2] Aliprantis, C.D. and Burkinshaw, O., On weakly compact operators on Banach lattices. Proc. Amer. Math. Soc. 83 (1981), no. 3, 573-578. MR0627695 (82j:47057)

[3] Aliprantis, C.D. and Burkinshaw, O., Dunford-Pettis operators on Banach lattices. Trans. Amer. Math. Soc. 274 (1982), no. 1, 227-238. MR0670929 (84b:47045)

[4] Aliprantis, C.D. and Burkinshaw, O., Positive operators. Pure and Applied Mathematics, 119. Academic Press, Inc., Orlando, FL, 1985. MR0809372 (87h:47086)

[5] Aliprantis, C.D. and Burkinshaw, O., Locally solid Riesz spaces with applications to economics. Mathematical Surveys and Monographs, Vol. 105, American Mathematical Society, Providence, RI, 2003. MR2011364 (2005b:46010)

[6] Aqzzouz, B. and Nouira, R., Les opérateurs précompacts sur les treillis vectoriels localement convexes-solides. Sci. Math. Jpn., 57 (2003), no. 2, 279-256. MR1959985 (2003k:46001)

[7] Aqzzouz, B. and Nouira, R., Sur les opérateurs précompacts positifs. C. R. Math. Acad. Sc. Paris, 337, (2003), no. 8, 527-530. MR2017131 (2004h:46002)

[8] Dodds, P.G. and Fremlin, D.H., Compact operators on Banach lattices. Israel J. Math. 34 (1979), 287-320. MR0570888 (81g:47037)

[9] Flores, J. and Hernández, F. L. Domination by positive disjointly strictly singular operators. Proc. Amer. Math. Soc. 129 (2001), no. 7, 1979-1986. MR1825905 (2002b:47075)

[10] Flores, J. and Hernandez, F.L., Domination by positive strictly singular operators. J. London Math. Soc. no. 2, 66 (2002), 433-452. MR1920413 (2003k:47047)

[11] Flores, J. and Ruiz, C., Domination by positive narrow operators. Positivity 7 (2003), no. 4, 303-321. MR2017310 (2004k:47071)

[12] Fremlin, D.H., Riesz spaces with the order continuity property I. Proc. Cambr. Phil. Soc. 81 (1977), 31-42. MR0425572 (54:13526)

[13] Kalton, N.J. and Saab, P., Ideal properties of regular operators between Banach lattices. Illinois Journal of Math. 29 (1985), no. 3, 382-400. MR0786728(87a:47064)

[14] Robertson, A.P. and Robertson, W., Topological vector spaces. $2^{\text {nd }}$ ed, Cambridge University Press, London, 1973. MR0350361 (50:2854) 
[15] Wickstead, A.W., Extremal structure of cones of operators. Quart. J. Math. Oxford 32 (1981), no. 2, 239-253. MR0615198 (82i:47069)

[16] Wickstead, A.W., Converses for the Dodds-Fremlin and Kalton-Saab Theorems. Math. Proc. Camb. Phil. Soc. 120 (1996), 175-179. MR1373356 (96m:47067)

[17] Wickstead, A.W., Positive compact operators on Banach lattices: some loose ends. Positivity 4 (2000), 313-325. MR1797133 (2001k:47054)

[18] Zaanen, A.C., Riesz spaces II. North-Holland Publishing Company, 1983. MR0704021 (86b:46001)

Département de Mathématiques, Faculté des Sciences, Université Ibn Tofail, Equipe D'Analyse Fonctionnelle, B.P. 133, Kénitra, Morocco

E-mail address: baqzzouz@hotmail.com

Département de Mathématiques, Faculté des Sciences, Université Ibn Tofail, Equipe D’Analyse Fonctionnelle, B.P. 133, Kénitra, Morocco

Département de Mathématiques, Faculté des Sciences, Université Ibn Tofail, Equipe D'Analyse Fonctionnelle, B.P. 133, Kénitra, Morocco 